\title{
Checkerboard local density of states in striped domains pinned by vortices
}

\author{
Andersen, B.M.; Hedegård, P.; Bruus, Henrik
}

Published in:

Physical Review B Condensed Matter

Link to article, DOI:

10.1103/PhysRevB.67.134528

Publication date:

2003

Document Version

Publisher's PDF, also known as Version of record

Link back to DTU Orbit

Citation (APA):

Andersen, B. M., Hedegård, P., \& Bruus, H. (2003). Checkerboard local density of states in striped domains pinned by vortices. Physical Review B Condensed Matter, 67(13), 134528.

https://doi.org/10.1103/PhysRevB.67.134528

\section{General rights}

Copyright and moral rights for the publications made accessible in the public portal are retained by the authors and/or other copyright owners and it is a condition of accessing publications that users recognise and abide by the legal requirements associated with these rights.

- Users may download and print one copy of any publication from the public portal for the purpose of private study or research.

- You may not further distribute the material or use it for any profit-making activity or commercial gain

- You may freely distribute the URL identifying the publication in the public portal

If you believe that this document breaches copyright please contact us providing details, and we will remove access to the work immediately and investigate your claim. 


\title{
Checkerboard local density of states in striped domains pinned by vortices
}

\author{
Brian Møller Andersen, ${ }^{1}$ Per Hedegård, ${ }^{1}$ and Henrik Bruus ${ }^{2}$ \\ ${ }^{1}$ Orsted Laboratory, Niels Bohr Institute for APG, Universitetsparken 5, DK-2100 Copenhagen Ф, Denmark \\ ${ }^{2}$ Mikroelektronik Centret, Technical University of Denmark, DK-2800 Lyngby, Denmark \\ (Received 3 September 2002; published 30 April 2003)
}

\begin{abstract}
We discuss recent elastic neutron scattering and scanning tunneling experiments on high- $T_{c}$ cuprates exposed to an applied magnetic field. Antiferromagnetic vortex cores operating as pinning centers for surrounding stripes is qualitatively consistent with the neutron data provided the stripes have the antiphase modulation. Within a Green's function formalism we study the low energy electronic structure around the vortices and find that besides the dispersive quantum interference there exists a non-dispersive checkerboard interference pattern consistent with recent scanning tunneling measurements. Thus both experiments can be explained from the physics of a single $\mathrm{CuO}_{2}$ plane.
\end{abstract}

DOI: 10.1103/PhysRevB.67.134528

PACS number(s): 74.72.-h, 74.25.Ha, 74.25.Jb, 72.10.Fk

The competing orders in high- $T_{c}$ cuprates remain a strong candidate for explaining some of the unusual features of these doped Mott insulators. ${ }^{1-5}$ The competition between superconducting order and antiferromagnetic order has recently attracted a large amount of both experimental and theoretical attention. In particular, experiments in the mixed state have revealed an interesting coexistence of these order parameters.

Elastic neutron scattering results on $\mathrm{La}_{2-x} \mathrm{Sr}_{x} \mathrm{CuO}_{2}(x$ $=0.10$ ) have shown that the intensity of the incommensurate peaks in the superconducting phase is considerably increased when a large magnetic field is applied perpendicular to the $\mathrm{CuO}_{2}$ planes. ${ }^{6}$ This enhanced intensity corresponds to a spin density periodicity of eight lattice constants $8 a_{0}$ extending far outside the vortex cores. Similar results have been obtained for the related material $\mathrm{La}_{2} \mathrm{CuO}_{4+y} \cdot{ }^{7}$ Nuclear magnetic resonance (NMR) experiments have shown evidence of antiferromagnetism in and around the vortex cores of nearoptimally doped $\mathrm{Tl}_{2} \mathrm{Ba}_{2} \mathrm{CuO}_{6+\delta} \cdot{ }^{8}$ Furthermore, muon spin rotation measurements from the mixed state of $\mathrm{YBa}_{2} \mathrm{Cu}_{3} \mathrm{O}_{6.50}$ find static antiferromagnetism in the cores. ${ }^{9}$ Consistent with these findings scanning tunneling microscopy (STM) measurements performed on the surface of $\mathrm{YBa}_{2} \mathrm{Cu}_{3} \mathrm{O}_{7-\delta}$ and $\mathrm{Bi}_{2} \mathrm{Sr}_{2} \mathrm{CaCu}_{2} \mathrm{O}_{8+x}$ (Refs. 10 and 11) have revealed very low density of states inside the vortex cores. ${ }^{12-14}$ Thus, there is increasing evidence for antiferromagnetic correlations in the vortex cores of the under- and optimally-doped regime of the hole doped cuprates. More recent STM measurements of slightly overdoped $\mathrm{Bi}_{2} \mathrm{Sr}_{2} \mathrm{CaCu}_{2} \mathrm{O}_{8+x}$ have shown a checkerboard halo of the local density of states (LDOS) around the vortex cores. ${ }^{15}$ This LDOS modulation observed at low energy, $|\omega|$ $<12 \mathrm{meV}$, was found to have half the period of the spin density wave (SDW) observed by neutron scattering (i.e., four lattice constants $4 a_{0}$ ), and to be oriented along the crystal axes of the $\mathrm{Cu}-\mathrm{O}$ plane.

The neutron experiments have been analyzed within phenomenological models that assume a close proximity to a quantum phase transition between ordinary superconductivity and a phase with antiferromagnetism or a phase where superconductivity coexists with SDW and charge density wave (CDW) orders. ${ }^{5,16-18}$ In these models the suppression of the superconducting order inside the vortex cores allows the competing magnetic order to arise. Demler et al. ${ }^{17}$ found that around the vortices the circulating supercurrents can similarly weaken the superconductivity and induce a SDW.

The field-induced checkerboard LDOS pattern in the mixed state has been recently considered within the framework of several models. ${ }^{18-23}$ In this paper we add to the discussion by calculating the LDOS in regions where a $d$-wave superconductor has been perturbed by induced magnetism. First, however, we note that a checkerboard spin modulation is inconsistent with the elastic neutron scattering experiments by Lake et al. ${ }^{6}$ on $\mathrm{La}_{2-x} \mathrm{Sr}_{x} \mathrm{CuO}_{2}(x=0.10)$. For example, assuming that the checkerboard CDW is intrinsic to the $\mathrm{Cu}-\mathrm{O}$ planes where it gives rise to a static SDW checkerboard pattern [Fig. 1(a)], the expected neutron diffraction pattern is shown in Fig. 1(b) ${ }^{24}$ As is evident there is a $45^{\circ}$ rotation of the four main incommensurate peaks and a plaid pattern of the higher harmonics. The rotated incommensurability [with the correct absence of an increased signal at $(\pi, \pi)$ ] shows that this spin structure does not apply to LSCO for doping levels close to $x=0.10$. It is interesting to note that a rotation of the incommensurable peaks at low dopings $(x<0.055$, close the insulator-superconductor phase transition) has been observed in LSCO. ${ }^{25}$ However, there is no simple way to create an antiphase spin geometry without frustrating the spins at low dopings where droplets of charge (a)

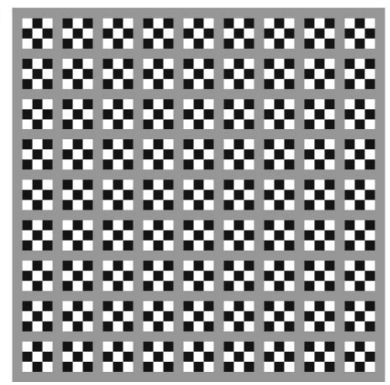

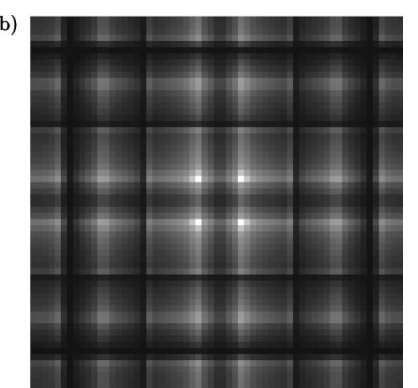

FIG. 1. (a) Real space picture of the spin structure in a checkerboard spin geometry. Black (white) represent spin up (down) while gray reveals the superconducting background. In order to simulate the induced incommensurability each island of antiferromagnetic spins is out of phase with its nearest neighbor. (b) Fourier spectrum of the spin checkerboard structure shown in (a). 
(a)
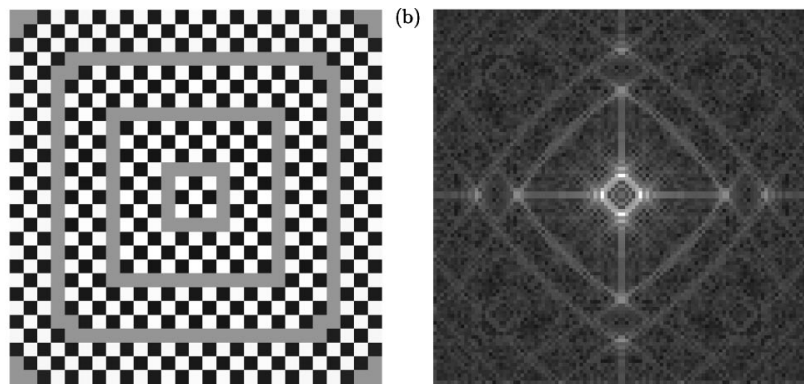

FIG. 2. (a) The idealized version of a real space spin configuration consistent with our physical picture. (b) Fourier spectrum of the spin density order from (a). Almost all the induced weight is located in the four incommensurable peaks.

in an antiferromagnetic background is the expected situation. ${ }^{26}$ However, this might be possible in the highly overdoped regime where the droplets have been inverted to separate magnetic islands. In that case a $45^{\circ}$ rotation of the incommensurable peaks would be consistent with a checkerboard spin pattern. In this light it would be very interesting to perform an experiment similar to that of Lake et al. ${ }^{6}$ on highly overdoped LSCO. In the case of a connected antiferromagnetic background one would also expect a large weight at $(\pi, \pi)$.

The physical picture we have in mind is presented in Fig. 2(a). In this real space picture an antiferromagnetic core (center) has pinned a number of surrounding stripes. This pinning effect of SDWs by magnetic vortex cores is a wellknown effect from numerical studies. ${ }^{21}$

Both experimentally ${ }^{28}$ and theoretically ${ }^{1,3,5,29}$ we expect an antiphase modulation of the induced antiferromagnetic ring domains. Indeed as seen in Fig. 2(b), the related diffraction pattern is qualitatively consistent with measurements by Lake et $a l .{ }^{6}$ of enhanced intensity at the incommensurate points.

Without an applied magnetic field, only disorder can produce a similar pinning effect of the fluctuating stripes. ${ }^{27}$ In addition to the creation of more pinning centers when applying a magnetic field, the single site impurities are expected to pin much weaker than the large "impurities" created by the flux lines. This is qualitatively consistent with the measurements by Lake et $a l .{ }^{6}$ of the temperature dependence of the increased magnetic signal for different magnetic field strengths.

This leads to the question of the electronic structure around extended magnetic perturbations in $d$-wave superconductors. The many experiments indicating coexistence of $d$-wave superconductivity and antiferromagnetism mentioned above motivate studies of simple models that enable one to calculate the LDOS in such regions.

The model Hamiltonian defined on a two-dimensional lattice is given by

$$
\begin{aligned}
\hat{H}^{0}= & -\sum_{\langle n, m\rangle \sigma} t_{n m} \hat{c}_{n \sigma}^{\dagger} \hat{c}_{m \sigma}-\mu \sum_{n \sigma} \hat{c}_{n \sigma}^{\dagger} \hat{c}_{n \sigma} \\
& +\sum_{\langle n, m\rangle}\left(\Delta_{n, m} \hat{c}_{n \uparrow}^{\dagger} \hat{c}_{m \downarrow}^{\dagger}+\text { H.c. }\right),
\end{aligned}
$$

$$
\hat{H}^{i n t}=\sum_{n} M_{n}\left(\hat{c}_{n \uparrow}^{\dagger} \hat{c}_{n \uparrow}-\hat{c}_{n \downarrow}^{\dagger} \hat{c}_{n \downarrow}\right)
$$

where $\hat{c}_{n \sigma}^{\dagger}$ creates an electron with spin $\sigma$ at site $n$ and $\mu$ is the chemical potential. The staggering is included in $M_{n}$ $=(-1)^{\mathbf{n}} M$. The strength of the antiferromagnetic and superconducting coupling is given by $M$ and $\Delta$, respectively.

The Hamiltonian $\hat{H}^{0}+\hat{H}^{i n t}$ is a simple mean-field lattice model to describe the phenomenology of the coexistence of $d$-wave superconducting and antiferromagnetic regions. This approach is similar to the starting point of many recent Bogoliubov-de Gennes calculations. ${ }^{12,13,21}$ The Hamiltonian in Eqs. (1) and (2) can be viewed as the mean-field Hamiltonian of a $t-U-V$ Hubbard model, where the nearest neighbor attraction $V$ gives rise to the $d$-wave superconductivity. In contrast the on-site Coulomb repulsion $U$ only causes the antiferromagnetism. In this paper we do not diagonalize $\hat{H}$ in the Bogoliubov-de Gennes scheme since such lattice calculations require unrealistically large gap $\Delta$ and magnetic field values. Instead we solve the Dyson equation exactly by inverting a large matrix. This approach has previously been utilized extensively to study various short-ranged impurity effects in superconductors, ${ }^{33}$ but can also be used for extended perturbations embedded in a $\hat{G}_{0}$ medium. Here $\hat{G}_{0}$ is the Green's function of the parent medium, in this case a $d$-wave BCS superconductor. This Green's function is given by

$$
\hat{G}_{0}^{-1}(\mathbf{p}, \omega)=(\omega+i \delta) \tau_{0}-\xi_{\mathbf{p}} \tau_{3}-\Delta_{\mathbf{p}} \tau_{1},
$$

where $\tau_{\nu}$ denote the Pauli matrices in Nambu space and the gap function $\Delta_{\mathbf{p}}=\left(\Delta_{0} / 2\right)\left[\cos \left(p_{x}\right)-\cos \left(p_{y}\right)\right]$. The lattice constant $a_{0}$ is set to unity and $\xi_{\mathbf{p}}=\epsilon_{\mathbf{p}}-\mu$ with

$$
\epsilon_{\mathbf{p}}=-2 t\left[\cos \left(p_{x}\right)+\cos \left(p_{y}\right)\right]-4 t^{\prime}\left[\cos \left(p_{x}\right) \cos \left(p_{y}\right)\right] .
$$

Here $t\left(t^{\prime}\right)$ refers to the nearest (next-nearest) neighbor hopping integral and $\mu$ is the chemical potential. We perform the two-dimensional Fourier transform of $G_{0}(\mathbf{p}, \omega)$ numerically by utilizing a real space lattice of $1000 \times 1000$ sites and a quasiparticle energy broadning of $\delta=1.0 \mathrm{meV}$.

To simulate the situation around optimal doping of the hole doped cuprates the following parameters are chosen: $t$ $=300 \mathrm{meV}, \quad t^{\prime}=-120 \mathrm{meV}, \quad \Delta_{0}=25 \mathrm{meV}, \quad$ and $\mu$ $=-354 \mathrm{meV}$. When the real space domain affected by $H^{i n t}$ involves a finite number of lattice sites $N \times N$ we can solve the Dyson equation exactly to find the full Greens function. Writing the Dyson equation in terms of real-space (and Nambu) matrices it becomes

$$
\underline{\underline{G}}(\omega)=\underline{\underline{G}}^{0}(\omega)\left[\underline{\underline{1}}-\underline{\underline{H}}^{i n t} \underline{\underline{G}}^{0}(\omega)\right]^{-1}
$$

The size of the matrix $\left[\underline{\underline{1}}-\underline{\underline{H}}^{i n t} \underline{\underline{G}}^{0}(\omega)\right]$ is given by $(d$ $\left.\times N^{2}\right) \times\left(d \times N^{2}\right)$, where $d$ is an integer equal to the number of components in the Nambu particle-hole spinor and $N$ denotes the total number of lattice sites affected by the magnetic perturbation. Therefore a real-space lattice with 25 $\times 25$ sites affected by perturbations results in a $1250 \times 1250$ matrix to being inverted. 

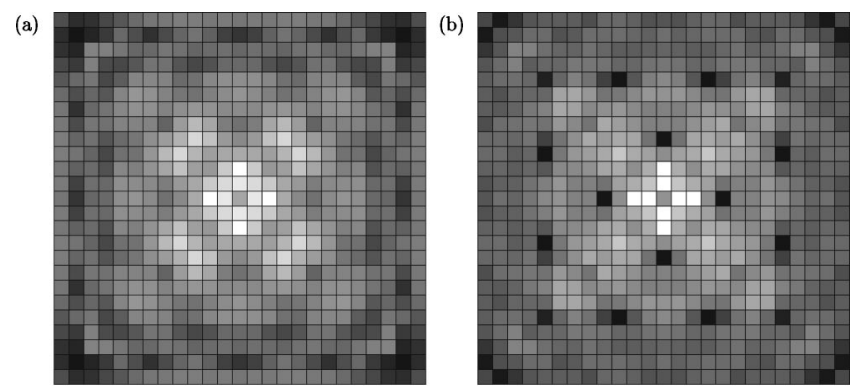

(c)

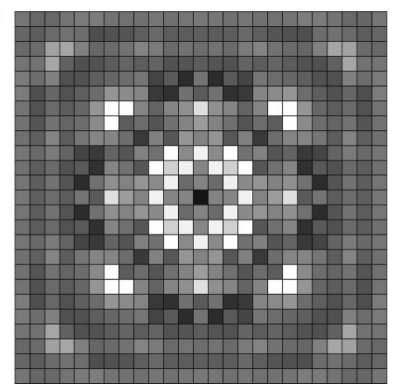

(d)

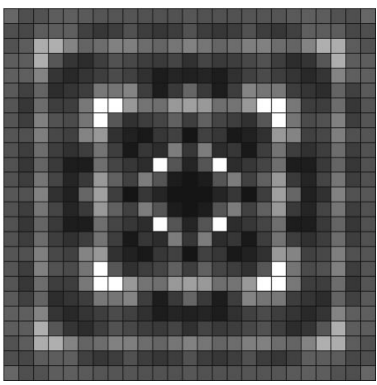

(a)

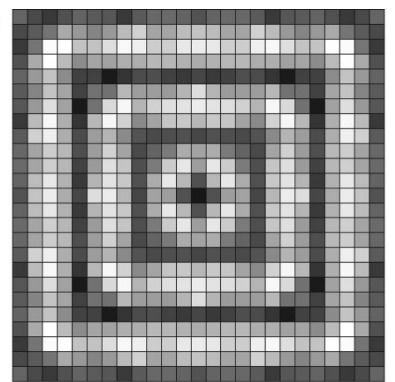

(c)

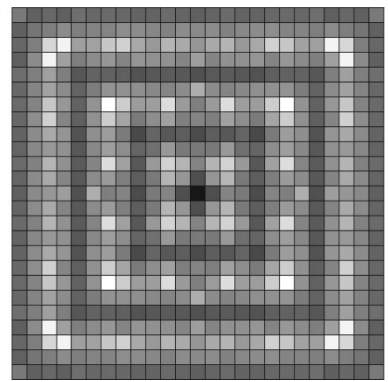

(b)

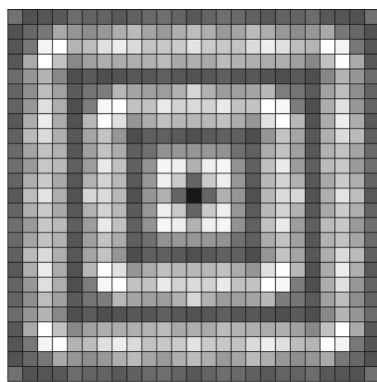

(d)

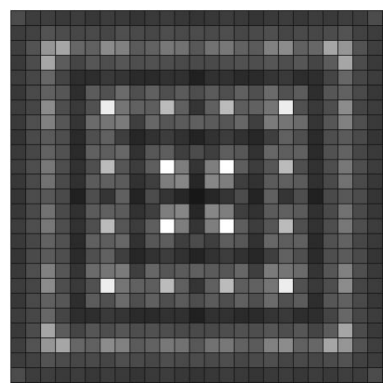

FIG. 3. Real-space LDOS summed from $-12 \mathrm{meV}$ to +12 $\mathrm{meV}$ for (a) $M=35 \mathrm{meV}$, (b) $M=100 \mathrm{meV}$, (c) $M=200 \mathrm{meV}$, and (d) $M=300 \mathrm{meV}$.

Knowing the full Greens function we obtain the LDOS $\rho(\mathbf{r}, \omega)=-(1 / \pi) \operatorname{Im}\left[G_{11}(\mathbf{r}, \omega)+G_{22}(\mathbf{r},-\omega)\right]$, which is proportional to the differential conductance measured in the STM experiments.

We have checked that the above approach reproduces the expected LDOS for unitary nonmagnetic impurities in $d$-wave superconductors. ${ }^{30}$ Also in this one-impurity case we reproduce the constant-energy LDOS maps recently calculated by Wang and Lee. ${ }^{31,32}$

Motivated by the qualitative agreement of the spin structure in Fig. 2(a) with the neutron data, we assume that this represents the induced magnetism around the vortices and calculate the LDOS in this striped environment. To this end we simply restrict the sum in Eq. (2) to include the sites within these magnetic regions. The system is depicted in Fig. 2(a) where the gray background reveals the underlying superconducting state. Again the black (white) squares correspond to the sites affected by the staggered magnetic perturbation.

Figures 3 and 4 show real-space maps of the LDOS summed over a small energy window from -12 to $+12 \mathrm{meV}$ in intervals of $1 \mathrm{meV}$ for different strengths of the antiferromagnetic perturbation $M$. The vortex center is located in the center of the images. Figure 3 (4) is calculated with (without) the antiphase modulation of the adjacent stripes. Thus the spin configuration of Fig. 2(a) corresponds to the images in Fig. 3. The clear difference between the LDOS images of Figs. 3 and 4 reveals that the STM technique can be used to determine this phase relation. It is clearly seen from both Figs. 3 and 4 that the low energy LDOS structure eventually becomes ringshaped as the magnitude of $M$ increases. In this limit the pinned stripes operate as steep potential walls. Figures 3(a) and 3(b) seem to display the closest resemblence to the experimental data ${ }^{15}$ which indicates that the induced

FIG. 4. Real-space LDOS summed from -12 to $+12 \mathrm{meV}$ for (a) $M=35 \mathrm{meV}$, (b) $M=100 \mathrm{meV}$, (c) $M=200 \mathrm{meV}$, and (d) $M$ $=300 \mathrm{meV}$.

magnetism is very weak. In Fig. 5 we show the Fourier transform of several constant energy LDOS images for $M$ $=100 \mathrm{meV}$ with the antiphase spin modulation included. In these figures the Fourier component $\mathbf{q}=0$ is located at the center. The detailed energy dependence of these images is caused by quasiparticle interference effects as pointed out by Wang and Lee ${ }^{31}$ in the case of a single impurity.

The dispersive features of the images presented in Fig. 5 are dependent on the microscopic parameters and the associated Fermi surface. However, it is also evident that the ring-

(a)

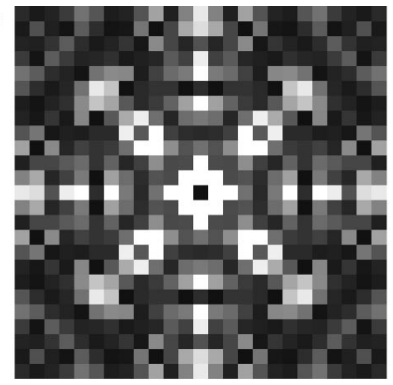

(c)

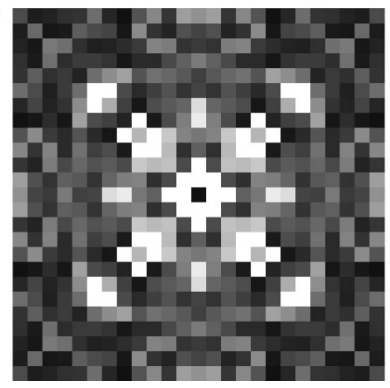

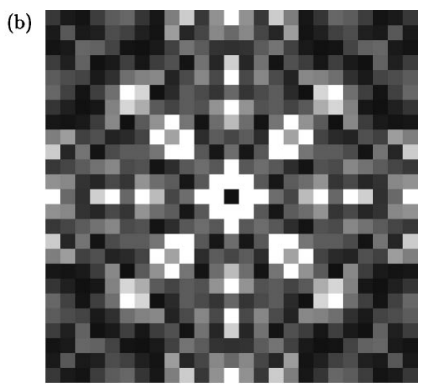

(d)

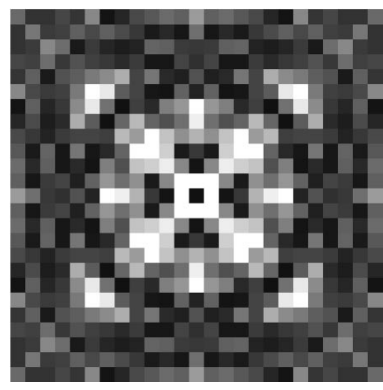

FIG. 5. Fourier images of the constant energy LDOS maps for $M=100 \mathrm{meV}$ and (a) $\omega=3 \mathrm{meV}$, (b) $\omega=6 \mathrm{meV}$, (c) $\omega=9 \mathrm{meV}$, and (d) $\omega=12 \mathrm{meV}$. 
shaped stripes surrounding the vortex cores give rise to nondispersive intensity around $\mathbf{q}=\left(2 \pi / a_{0}\right)( \pm 1 / 4,0)$ and $\mathbf{q}$ $=\left(2 \pi / a_{0}\right)(0, \pm 1 / 4)$. This in turn leads to the checkerboard pattern in the low energy sums of the LDOS displayed in Figs. 3 and 4 whereas the dispersive features fade away in these summed LDOS images. ${ }^{27}$

We have confirmed this fact by identifying similar nondispersive features in the LDOS around configurations with different periodicities. For instance, a structure with $2 a_{0}$ charge periodicity leads to a nondispersive intensity around $\mathbf{q}=\left(2 \pi / a_{0}\right)( \pm 1 / 2,0)$ and $\mathbf{q}=\left(2 \pi / a_{0}\right)(0, \pm 1 / 2)$.

In the above calculation we have not yet included the Doppler shift from the circulating supercurrents or the gap suppression close to the vortex core. As pointed out by Polkovnikov et al. ${ }^{18}$ the former effect is not expected to produce significant changes of the four-period modulations. As for the latter we have checked that a gap suppression only leads to minor quantitative changes in the dispersive part of the LDOS. Finally, Podolsky et al. ${ }^{34}$ discussed scenarios of weak translational symmetry breaking and found that in order to explain quantitatively the zero-field STM results by Howald et $a l .{ }^{27}$ one needs to include dimerization, the modulation of the electron hopping. This dimerization will also produce quantitative changes, but not alter the qualitative conclusion that pinned stripes produce checkerboard LDOS.

In summary, we have discussed the phenomenology of a simple physical picture of pinned stripes around vortex cores which are forced to be antiferromagnetic by an applied magnetic field. The induction of magnetic striped race tracks around the core is consistent with the neutron diffraction spectra observed on LSCO with a doping level near $x$ $=0.10$. As expected this is only true if the stripes are out of phase with their neighbors in the usual sense. In materials where a checkerboard spin pattern is relevant (possibly $\mathrm{Bi} 2212$ or overdoped LSCO), we show that a $45^{\circ}$ rotation of the main incommensurable peaks is to be expected. Finally we studied the electronic structure around the vortices and identified a non-dispersive feature in the LDOS arising from the induced static antiferromagnetism. This feature gives rise to the checkerboard LDOS observed experimentally by Hoffman et al. ${ }^{15}$ Thus both the STM measurements and the enhanced intensity of the incommensurable peaks observed by neutron diffraction can be ascribed to the phenomena of a single $\mathrm{CuO}_{2}$ plane.

Support by the Danish Natural Science Research Council, Ole Rømer Grant No. 9600548, is acknowledged.
${ }^{1}$ J. Zaanan and O. Gunnarson, Phys. Rev. B 40, 7391 (1989).

${ }^{2}$ V.J. Emery and S.A. Kivelson, Physica C 209, 597 (1993).

${ }^{3}$ S.A. Kivelson, E. Fradkin, and V.J. Emery, Nature (London) 393, 550 (1998); V.J. Emery, S.A. Kivelson, and J.M. Tranquada, Proc. Natl. Acad. Sci. U.S.A. 96, 8814 (1999).

${ }^{4}$ S.R. White and D.J. Scalapino, Phys. Rev. Lett. 81, 3227 (1998).

${ }^{5}$ M. Vojta and S. Sachdev, Phys. Rev. Lett. 83, 3916 (1999).

${ }^{6}$ B. Lake, H.M. Rønnow, N.B. Christensen, G. Aeppli, K. Lefmann, D.F. McMorrow, P. Vorderwisch, P. Smeibidl, N. Mangkorntong, T. Sasagawa, M. Nohara, H. Takagi, and T.E. Mason, Nature (London) 415, 299 (2002).

${ }^{7}$ B. Khaykovich, Y.S. Lee, R.W. Erwin, S.-H. Lee, S. Wakimoto, K.J. Thomas, M.A. Kastner, and R.J. Birgeneau, Phys. Rev. B 66, 014528 (2002).

${ }^{8}$ K. Kakuyanagi, K. Kumagai, Y. Matsuda, and M. Hasegawa, cond-mat/0206362 (unpublished).

${ }^{9}$ R.I. Miller, R.F. Kiefl, J.H. Brewer, J.E. Sonier, J. Chakhalian, S. Dunsiger, G.D. Morris, A.N. Price, D.A. Bonn, W.H. Hardy, and R. Liang, Phys. Rev. Lett. 88, 137002 (2002).

${ }^{10}$ I. Maggio-Aprile, Ch. Renner, A. Erb, E. Walker, and $\emptyset$. Fischer, Phys. Rev. Lett. 75, 2754 (1995); C.Renner, B. Revaz, K. Kadowaki, I. Maggio-Aprile, and Ø. Fischer, Phys. Rev. Lett. 80, 3606 (1998).

${ }^{11}$ S.H. Pan, E.W. Hudson, A.K. Gupta, K.-W. Ng, H. Eisaki, S. Uchida, and J.C. Davis, Phys. Rev. Lett. 85, 1536 (2000).

${ }^{12}$ B.M. Andersen, H. Bruus, and P. Hedegård, Phys. Rev. B 61, 6298 (2000).

${ }^{13}$ J-X. Zhu and C.S. Ting, Phys. Rev. Lett. 87, 147002 (2001).

${ }^{14}$ A. Ghosal, C. Kallin, and A.J. Berlinsky, Phys. Rev. B 66, 214502 (2002).

${ }^{15}$ J.E. Hoffman, E.W. Hudson, K.M. Lang, V. Madhavan, H. Eisaki,
S. Uchida, and J.C. Davis, Science (Washington, DC, U.S.) 295, 466 (2002).

${ }^{16}$ D.P. Arovas, A.J. Berlinsky, C. Kallin, and S.-C. Zhang, Phys. Rev. Lett. 79, 2871 (1997).

${ }^{17}$ E. Demler, S. Sachdev, and Y. Zhang, Phys. Rev. Lett. 87, 067202 (2001).

${ }^{18}$ A. Polkovnikov, M. Vojta, and S. Sachdev, Phys. Rev. B 65, 220509 (2002).

${ }^{19}$ H.D. Chen, J.-P. Hu, S. Capponi, E. Arrigoni, and S.-C. Zhang, Phys. Rev. Lett. 89, 137004 (2002).

${ }^{20}$ A. Polkovnikov, S. Sachdev, M. Vojta, cond-mat/0208334 (unpublished).

${ }^{21}$ Y. Chen and C.S. Ting, Phys. Rev. B 65, 180513 (2002); Y. Chen, H.Y. Chen, and C.S. Ting, cond-mat/0203283 (unpublished).

${ }^{22}$ M. Franz, D.E. Sheeny, and Z. Tesanovic, Phys. Rev. Lett. 88, 257005 (2002).

${ }^{23}$ J-X. Zhu, I. Martin, and A.R. Bishop, Phys. Rev. Lett. 89, 067003 (2002).

${ }^{24}$ B.M. Andersen and P. Hedegård, cond-mat/0206502 (unpublished).

${ }^{25}$ S. Wakimoto, G. Shirane, Y. Endoh, K. Hirota, S. Ueki, K. Yamada, R.J. Birgeneau, M.A. Kastner, Y.S. Lee, P.M. Gehring, and S.H. Lee, Phys. Rev. B 60, R769 (1999).

${ }^{26}$ M. Veilette, Y.B. Bazaliy, A.J. Berlinsky, and C. Kallin, Phys. Rev. Lett. 83, 2413 (1999).

${ }^{27}$ C. Howald, H. Eisaki, N. Kaneko, and A. Kapitulnik, cond-mat/0201546 (unpublished); C. Howald, H. Eisaki, N. Kaneko, M. Greven, and A. Kapitulnik, cond-mat/0208442 (unpublished).

${ }^{28}$ J.M. Tranquada, B.J. Sternlieb, J.D. Axe, Y. Nakamura, and S. Uchida, Nature (London) 375, 561 (2002). 
${ }^{29}$ O. Zachar, S.A. Kivelson, and V.J. Emery, Phys. Rev. B 57, 1422 (1998).

${ }^{30}$ A.V. Balatsky, M.I. Salkola, and A. Rosengren, Phys. Rev. B 51, 15547 (1995).

${ }^{31}$ Q.-H. Wang and D.-H. Lee, Phys. Rev. B 67, 020511 (2003).

${ }^{32}$ Naturally the full agreement is only obtained when using the same quasiparticle energy $\xi_{\mathbf{p}}$ as Wang and Lee (Ref. 31).

${ }^{33}$ M.E. Flatte and J.M. Byers, Phys. Rev. Lett. 80, 4546 (1998); Phys. Rev. B 56, 11213 (1997).

${ }^{34}$ D. Podolsky, E. Demler, K. Damle, and B.I. Halperin, cond-mat/0204011 (unpublished). 